\title{
Synthesis of Ni Nanopowders Using an EHA System
}

\author{
Desheng Ai and Shinhoo Kang \\ School of Materials Science \& Engineering, Seoul National University, Seoul 151-742, Korea
}

Nickel nanopowders were synthesized using an ethylene glycol-hydrazine-ammonia (EHA) system. Based on X-ray diffraction data, the resulting powders were identified as pure crystalline nickel with an average crystal size of $\sim 20 \mathrm{~nm}$. The mean diameter of the Ni powders decreased as the amount of hydrazine used was increased. A small amount of an $\mathrm{AgNO}_{3}$ solution, a nucleating agent, was found to facilitate the formation of the nanopowders. Ethylene glycol was found to be effective in preventing the nanopowders from becoming agglomerated. [doi: $10.2320 /$ matertrans.47.2056]

(Received November 30, 2005; Accepted June 23, 2006; Published August 15, 2006)

Keywords: nanopowders, ethylene glycol-hydrazine-ammonia system, nickel, powder synthesis

\section{Introduction}

The preparation of dispersed, nano-size powders or clusters has been a subject of extensive investigation over the past two decades, because of their unusual properties and potential applications. ${ }^{1)}$ Fine Ni powders are required for high-tech applications in alkaline rechargeable batteries, magnetic recording media and for use as chemical catalysts. ${ }^{2)}$ A variety of methods have been reported for the synthesis of ultra-fine nickel powders and these include physical methods such as ball milling, pulsed electro-deposition (PED), thermal plasma, gas deposition methods (GDM), $\gamma$-radiation, ${ }^{3)}$ and chemical methods. Some of the chemical methods involve sono-chemical decomposition, ${ }^{4)}$ chemical reduction, ${ }^{5)}$ a microwave hydrothermal method, ${ }^{6)}$ mechanical alloying, mechano-chemical processing and a polyol process. $^{7,8)}$ Of these methods, chemical reduction in an aqueous solution is particularly well-suited to the synthesis of nano or ultra-fine nickel powders. ${ }^{9-11)}$ When this method is used, chemical control over the size and dispersed property of the powders is a challenge.

In order to develop a simple method for the synthesis of nickel nanopowders, Degen et al. ${ }^{12-15)}$ used nickel acetate, which was then dissolved in special organic solvents to produce nickel powders. There are several chemicals involved in synthesizing nickel nanopowder via wet chemistry. The system should have a nucleating agent for the precipitation, a particle size inhibitor and surfactant to control the agglomeration. In this study, a process for the production of nickel nanopowders using an EHA system is described. The system, which is composed of ethylene glycol, hydrazine and ammonia water, is referred to herein as the EHA system. EHA system is designed considering the characteristics of the solvent. For this approach ethylene glycol was used as a solvent without surfactant. A small amount of $\mathrm{AgNO}_{3}{ }^{16)}$ solution and aqueous ammonia were used as a nucleating agent and a $\mathrm{pH}$ controller, respectively. We will discuss the role of EHA system in forming nanocrystalline nickel.

\section{Experimental}

Commercially available nickel chloride, $\mathrm{NiCl}_{2}$, (Duksan
Pure Chem. Co. Ltd., Korea) was used as the starting material with hydrazine monohydrate, $\mathrm{N}_{2} \mathrm{H}_{4} \cdot \mathrm{H}_{2} \mathrm{O}$, (Duksan Pure Chem. Co. Ltd., Korea) as the reducing agent. An aqueous solution of ammonia, $\mathrm{NH}_{4} \mathrm{OH}$, (Duksan Pure Chem. Co. Ltd., Korea) and ethylene glycol, $\mathrm{HOCH}_{2} \mathrm{CH}_{2} \mathrm{OH}$, (Samchun Pure Chem. Ind. Ltd., Korea) were used as a $\mathrm{pH}$ modifier and solvent, respectively. A $0.1 \mathrm{~mol} / \mathrm{L}$ silver nitrate solution, $\mathrm{AgNO}_{3}$, (Shinyo Pure Chem. Co. Ltd., Japan) was employed as the nucleating agent.

A suitable amount of nickel chloride was dissolved directly in ethylene glycol and appropriate amounts of hydrazine and aqueous ammonia were sequentially added to the solution. When the temperature of the mixture reached $85^{\circ} \mathrm{C}$, a small amount of silver nitrate solution was added. The resulting suspension was stirred for $20 \mathrm{~min}$ and then allowed to age for an additional $30 \mathrm{~min}$, during which the formation of Ni powders occurred.

The mixture was filtered and the residue was washed with ethanol. After centrifugal filtration on a Centrifuger MF 80 (Hanil Sci. Ind., Korea) at a speed of 3000 RPM for $5 \mathrm{~min}$, the sample was dried at $50^{\circ} \mathrm{C}$ for a period of $5 \mathrm{~h}$ to give solid nickel powders. The reduction reaction can be expressed generally as shown below. ${ }^{9)}$

$$
2 \mathrm{Ni}^{2+}+\mathrm{N}_{2} \mathrm{H}_{4}+4 \mathrm{OH}^{-}=2 \mathrm{Ni} \downarrow+\mathrm{N}_{2} \uparrow+4 \mathrm{H}_{2} \mathrm{O}
$$

Analyses were performed by transmission electron microscopy (TEM), scanning electron microscopy (SEM) and X-ray diffraction (XRD) to determine the particle size, size distribution and particle configuration and to identify the present phase.

\section{Results}

\subsection{Powder characterization}

Figure 1 shows an XRD pattern of the Ni powders obtained in this study. Three characteristic peaks for nickel ( $2 \theta=44.5,51.8$ and $76.4^{\circ}$ ) were observed, corresponding to the (111), (200) and (220) planes of $\mathrm{Ni}$, respectively. This indicates that the powder is pure nickel with a face-centered cubic (fcc) crystal structure. The crystal size was estimated to be $\sim 18.5 \mathrm{~nm}$ from this result using Scherrer's formula. For the analysis it was assumed that there is no strain in the powder after synthesis. 


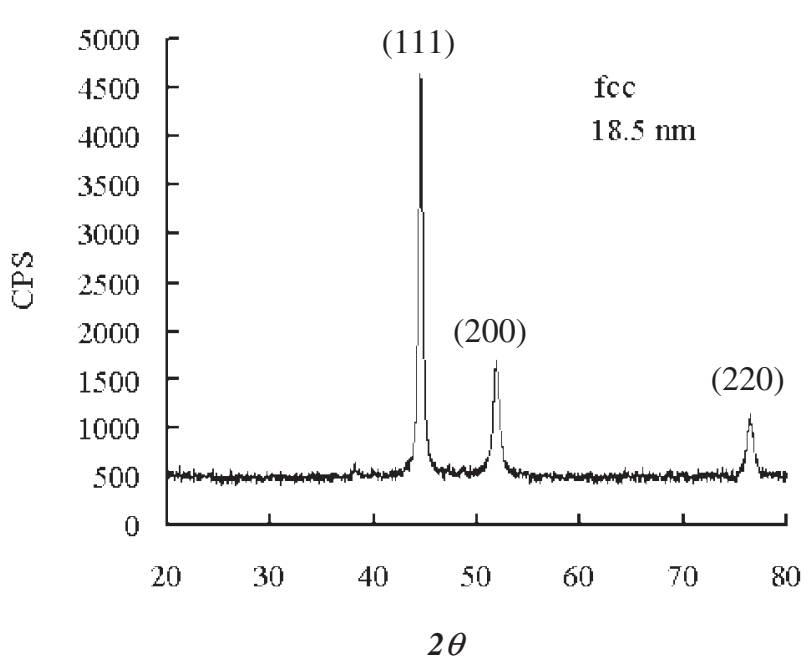

Fig. 1 XRD pattern of nickel nanopowders. $\left[\mathrm{Ni}^{2+}\right]=0.05 \mathrm{~mol} / \mathrm{L}$; $\left[\mathrm{N}_{2} \mathrm{H}_{5} \mathrm{OH}\right]=0.5 \mathrm{~mol} / \mathrm{L}$.
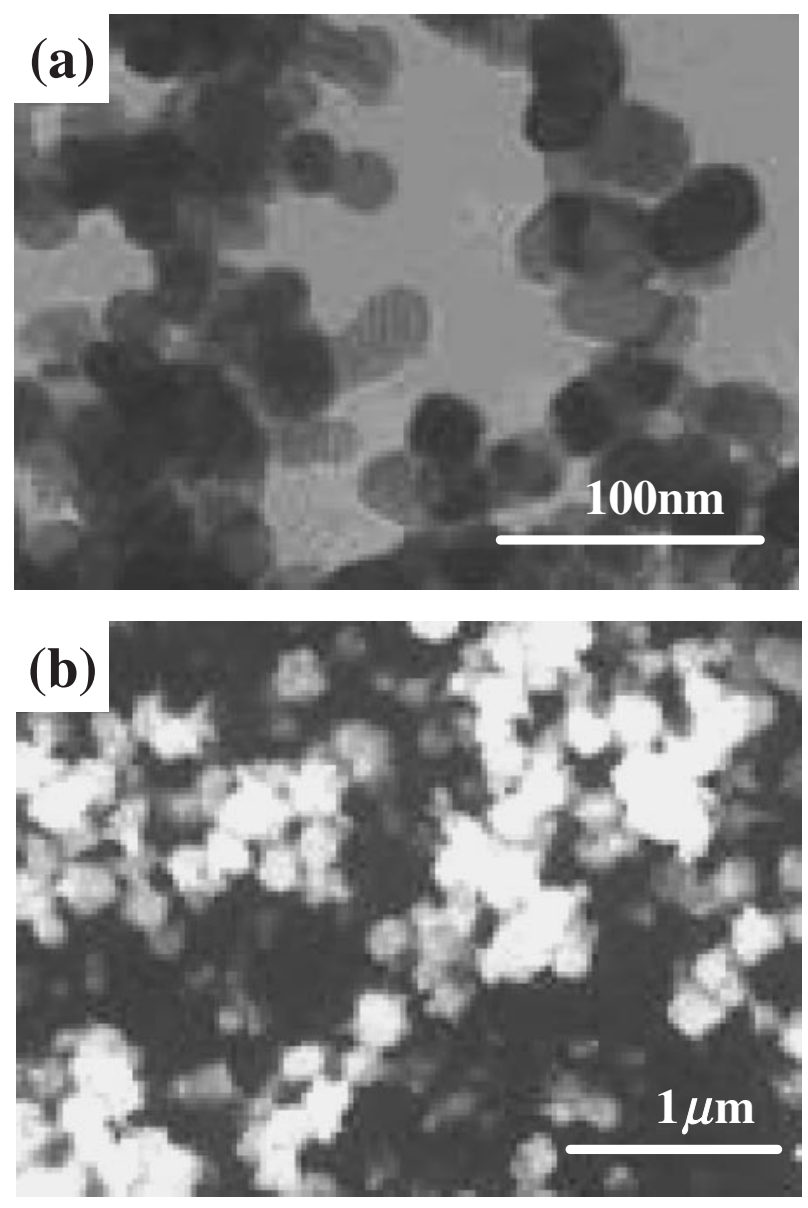

Fig. 2 Morphology of nickel nanopowders. $\left[\mathrm{Ni}^{2+}\right]=0.05 \mathrm{~mol} / \mathrm{L} ;\left[\mathrm{N}_{2} \mathrm{H}_{5}{ }^{-}\right.$ $\mathrm{OH}]=0.5 \mathrm{~mol} / \mathrm{L}$. (a) Transmission electron microscopy, (b) Scanning electron microscopy.

Figure 2 shows typical TEM and SEM micrographs at different magnifications for the nickel nanopowders prepared in this study. The TEM micrograph shows that Ni particles are spherical or lenticular in form, with a mean diameter of $\sim 20 \mathrm{~nm}$. This size is consistent with the result estimated by

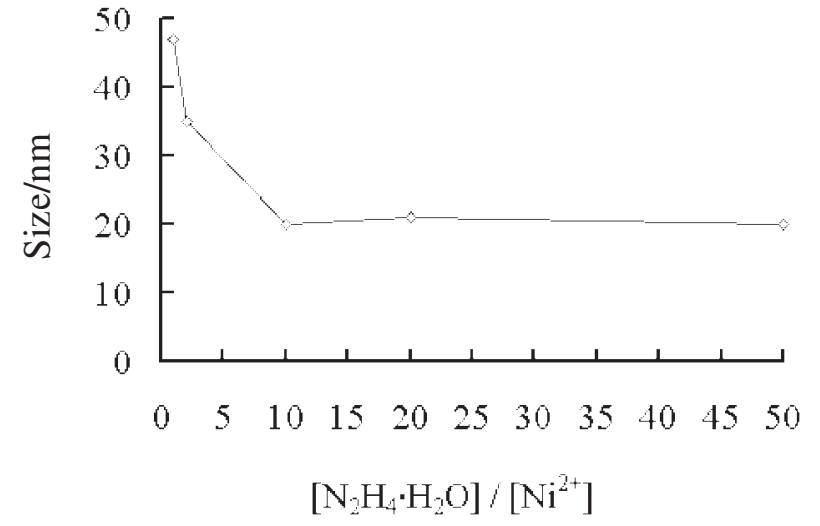

Fig. 3 Effect of $\left[\mathrm{N}_{2} \mathrm{H}_{4} \cdot \mathrm{H}_{2} \mathrm{O}\right]$ and $\left[\mathrm{Ni}^{2+}\right]$ ratio on the size of nickel powders.

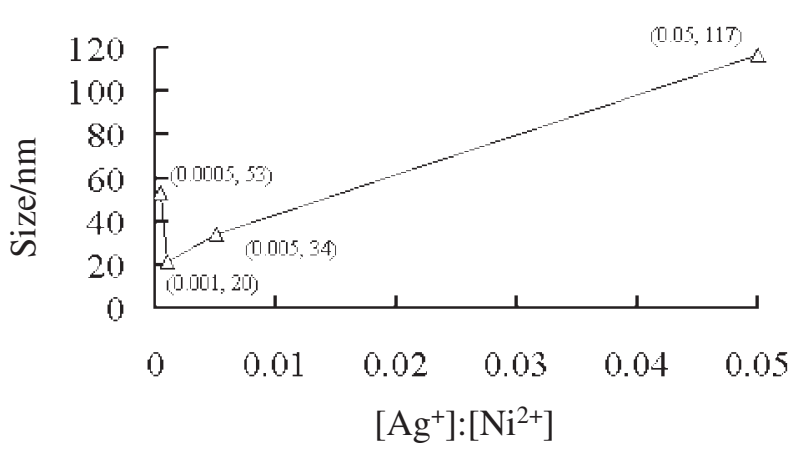

Fig. 4 Effect of $\mathrm{Ag}^{+}$concentrations on the size of nickel powders. $\left[\mathrm{Ni}^{2+}\right]=0.05 \mathrm{~mol} / \mathrm{L} ;\left[\mathrm{N}_{2} \mathrm{H}_{5} \mathrm{OH}\right]=0.5 \mathrm{~mol} / \mathrm{L}$.

Scherrer's formula. Figure 2(b) shows that the powders are in the form of loose agglomerates of about $100-200 \mathrm{~nm}$ in size. Compared to other magnetic nanopowders, the synthesized $\mathrm{Ni}$ powder is rather well-dispersed with a loose agglomeration.

It is not easy to disperse such nanopowders completely due to the magnetic properties of nickel and the high surface reactivity of the nanopowder. ${ }^{17)} \mathrm{A}$ protective polymer is commonly used in wet chemical methods to inhibit particle agglomeration. In this study well-dispersed nanopowders were obtained using ethylene glycol even without a protective polymer. This represents a significant advantage in the handling of nano Ni powders.

\subsection{Effect of parameters on particle size}

The effect of $\left[\mathrm{N}_{2} \mathrm{H}_{4} \cdot \mathrm{H}_{2} \mathrm{O}\right] /\left[\mathrm{Ni}^{2+}\right]$ and the concentration of the nucleating agent, $\mathrm{Ag}^{+}$, on the size of nickel nanopowders was investigated and the results are summarized in Figs. 3 and 4, respectively. As shown in Fig. 3, with increasing ratio of $\left[\mathrm{N}_{2} \mathrm{H}_{4} \cdot \mathrm{H}_{2} \mathrm{O}\right] /\left[\mathrm{Ni}^{2+}\right]$, the size decreased until the ratio reached $\sim 10$. Thereafter, the size remained unchanged. Based on the trend of that, the minimum size of particles that can be produced using a $\left[\mathrm{N}_{2} \mathrm{H}_{4} \cdot \mathrm{H}_{2} \mathrm{O}\right] /\left[\mathrm{Ni}^{2+}\right]$ ratio is in the range of $5-10 \mathrm{~nm}$.

The relationship between $\mathrm{Ag}^{+}$concentration and the size of the nickel powders is shown in Fig. 4. The presence of a nucleating agent was also found to be critical in the synthesis 
of Ni nanopowders. That is, when no nucleating agent was used, the reaction not only became sluggish, but the produced particles were coarse. After adding $\mathrm{AgNO}_{3}$ to the solution, a large number of $\mathrm{Ni}$ nuclei were formed, resulting in a smaller size powder. However, the optimum ratio of $\left[\mathrm{Ag}^{+}\right]$to $\left[\mathrm{Ni}^{2+}\right]$ should be around 0.001 to guarantee the smallest size. The addition of an excess of $\left[\mathrm{Ag}^{+}\right]$was detrimental to controlling powder size, resulting in particle coarsening. In addition, it led to $\mathrm{Ag}$ contamination of the $\mathrm{Ni}$ nanopowder. In a previous study, $\mathrm{PdCl}_{2} \cdot 2 \mathrm{H}_{2} \mathrm{O}$ or $\mathrm{H}_{2} \mathrm{PtCl}_{2} \cdot 6 \mathrm{H}_{2} \mathrm{O}$ was used as a nucleating agent. ${ }^{8)}$ Considering the cost, $\mathrm{AgNO}_{3}$ is more appropriate as a nucleating agent in practical applications.

\section{Discussion}

\subsection{Formation of nickel nanopowders}

The process by which $\mathrm{Ni}$ powder is formed was investigated by observing the color change of solution. A rapid color change from green to dark-blue occurred during the experiment. The sequence involved in the precipitation process can be described by eqs. (2), (3) and (4) below:

$$
\begin{aligned}
& \mathrm{Ni}^{2+}+3 \mathrm{HOCH}_{2} \mathrm{CH}_{2} \mathrm{OH} \rightarrow \mathrm{Ni}\left(\mathrm{HOCH}_{2} \mathrm{CH}_{2} \mathrm{OH}\right)_{3}{ }^{2+} \\
& \mathrm{Ni}\left(\mathrm{HOCH}_{2} \mathrm{CH}_{2} \mathrm{OH}\right)_{3}{ }^{2+}+6 \mathrm{~N}_{2} \mathrm{H}_{4} \\
& \quad \rightarrow \mathrm{Ni}\left(\mathrm{N}_{2} \mathrm{H}_{4}\right)_{6}{ }^{2+}+3 \mathrm{HOCH}_{2} \mathrm{CH}_{2} \mathrm{OH} \\
& \mathrm{Ni}\left(\mathrm{N}_{2} \mathrm{H}_{4}\right)_{6}{ }^{2+}+2 \mathrm{OH}^{-} \\
& \quad=\mathrm{Ni} \downarrow+5 \mathrm{~N}_{2} \mathrm{H}_{4}+2 \mathrm{H}_{2} \mathrm{O}+\mathrm{N}_{2} \uparrow+\mathrm{H}_{2} \uparrow
\end{aligned}
$$

When $\mathrm{NiCl}_{2} \cdot 6 \mathrm{H}_{2} \mathrm{O}$ is dissolved in ethylene glycol, a greencolored $\mathrm{Ni}\left(\mathrm{HOCH}_{2} \mathrm{CH}_{2} \mathrm{OH}\right)_{3}{ }^{2+}$ ion forms as in eq. 2. $\mathrm{Ni}\left(\mathrm{HOCH}_{2} \mathrm{CH}_{2} \mathrm{OH}\right)_{3}{ }^{2+}$ can be easily replaced by $\mathrm{Ni}\left(\mathrm{N}_{2}-\right.$ $\left.\mathrm{H}_{4}\right)_{6}{ }^{2+}$, which is dark-blue in color (eq. 3). $\mathrm{Ni}^{2+}$ is readily reduced by $\mathrm{N}_{2} \mathrm{H}_{4}$, forming $\mathrm{N}_{2} \mathrm{H}_{4} \cdot \mathrm{H}_{2} \mathrm{O}$ (eq. 4) and a $\mathrm{Ni}$ powder. In this process, $\mathrm{OH}^{-}$from the $\mathrm{NH}_{4} \mathrm{OH}$ resulted in the production of nanopowders. $\mathrm{NaOH}$ can also be used for the same purpose, but it leads to a significant coarsening of the Ni powders.

$\mathrm{Ni}$ precipitates were not observed when ethylene glycol was used without the addition of a sufficient amount of hydrazine. This indicates that hydrazine, rather than ethylene glycol, is necessary for the reduction of nickel ions. It was also found that the addition of $\mathrm{OH}^{-}$accelerates the formation of pure nickel nanopowders in ethylene glycol. ${ }^{14,16)}$ Otherwise, the process is slow.

Further, an elevated temperature and a trace of nucleating agent such as an $\mathrm{AgNO}_{3}$ solution accelerated the reaction rate. The formation of nickel nanopowders was complete within $15 \mathrm{~min}$ at $85^{\circ} \mathrm{C}$. At a temperature of $65^{\circ} \mathrm{C}$, the reaction required more than $3 \mathrm{~h}$ and would never be complete, if conducted at room temperature. Hence, the reaction temperature was fixed at $85^{\circ} \mathrm{C}$ in this work. An excessive amount of $\left[\mathrm{Ag}^{+}\right]$and high reaction temperature promote the growth of nuclei, resulting in a powder containing large sized particles.

\subsection{Powder agglomeration}

The presence of a protective polymer is known to reduce static charges among nano-particles. The reduced charge should lead to decrease the agglomeration for charged particles. ${ }^{18)}$ However, this work shows that the agglomeration of powders can be minimized. Well-dispersed nano-

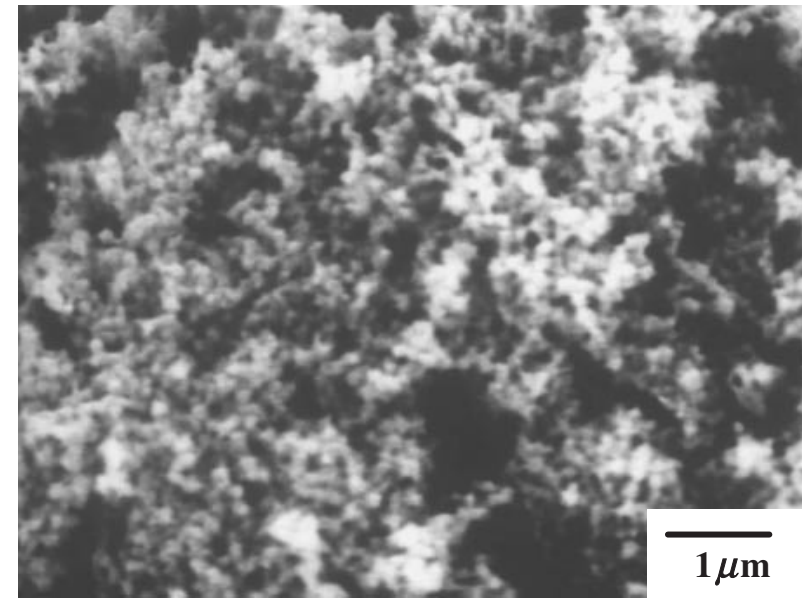

Fig. 5 Nickel nanopowders before drying: $\left[\mathrm{Ni}^{2+}\right]=0.05 \mathrm{~mol} / \mathrm{L} ;\left[\mathrm{N}_{2} \mathrm{H}_{5^{-}}\right.$ $\mathrm{OH}]=0.5 \mathrm{~mol} / \mathrm{L}$.

powders shown in Fig. 2 were obtained using ethylene glycol even without a protective polymer. This suggests that ethylene glycol played the role of a protective layer on the particle surface in the reaction. This might occur via interactions between $\mathrm{OH}$ groups and nickel atoms. ${ }^{16)}$

It is interesting to note the additional role of ethylene glycol. Figure 5 shows an SEM micrograph of nanopowders, after washing with ethanol. At this stage, the ethylene glycol was not completely removed by the washing process. Thus, the powders remained in a paste form. However, the powders became dispersed as shown in Fig. 2 when the ethylene glycol was evaporated at $50^{\circ} \mathrm{C}$. This indicates that a protective chemical, like ethylene glycol, might reduce the agglomeration by minimizing contact among the particles. That is known to be a cause of the static charge on fine particles.

When water was used in place of ethylene glycol, no pastry powder was produced. However, the size of the Ni particles was large due to the growth of the powders during the synthesis. Typically, it is difficult to completely disperse such nanopowders due to the magnetic properties of nickel and the high reactivity of the nanopowder surface. ${ }^{17)}$ However, the magnetic properties of nickel had no significant effect on the final nanopowders, when they were dispersed in ethylene glycol. This is a significant finding in the production of nanopowders.

\subsection{Effect of parameters on particle size}

The effect of $\left[\mathrm{N}_{2} \mathrm{H}_{4} \cdot \mathrm{H}_{2} \mathrm{O}\right] /\left[\mathrm{Ni}^{2+}\right]$ on the size of $\mathrm{Ni}$ particles can be attributed to the rate of reduction during the process. In wet chemistry, the growth of precipitates is usually faster than the nucleation process. Therefore, the nucleation process in the reaction is important in determinant of the size of the powders. Nuclei of powders start forming in ethylene glycol in the early stages of the reduction. In this work, at a low hydrazine concentration, $\left[\mathrm{N}_{2} \mathrm{H}_{4} \cdot \mathrm{H}_{2} \mathrm{O}\right] /$ $\left[\mathrm{Ni}^{2+}\right]<10$, the rate of reduction of nickel chloride was relatively slow and only a few nuclei were formed in the early stages of the reduction. As a result, powder growth was dominant, leading to the formation of large particles. 
However, with an increase in hydrazine concentration, the rate of reduction is enhanced, thus generating more nuclei as reported in elsewhere. ${ }^{14,16)}$ This leads to the formation of smaller nickel nanopowders, providing the $\left[\mathrm{N}_{2} \mathrm{H}_{4} \cdot \mathrm{H}_{2} \mathrm{O}\right] /$ $\left[\mathrm{Ni}^{2+}\right]$ ratio is $<10$. When the ratio of $\left[\mathrm{N}_{2} \mathrm{H}_{4} \cdot \mathrm{H}_{2} \mathrm{O}\right]$ to $\left[\mathrm{Ni}^{2+}\right]$ was sufficiently large, i.e., $\left[\mathrm{N}_{2} \mathrm{H}_{4} \cdot \mathrm{H}_{2} \mathrm{O}\right] /\left[\mathrm{Ni}^{2+}\right] \geq 10$, the rate of reduction reaches a maximum value and no further increase in nucleation rate takes place. The number of nuclei then remains constant with an increase in hydrazine concentration.

The addition of $\mathrm{AgNO}_{3}$ to the solution caused the formation of a number of $\mathrm{Ni}$ nuclei. The reason is that $\mathrm{Ag}^{+}$ is reduced rapidly and this facilitates the formation of nickel powders. If an excess of $\mathrm{AgNO}_{3}(>0.001 \mathrm{~mol} / \mathrm{L})$ is added, the nickel powders begin to coalesce, due to strong interactions among $\mathrm{Ag}, \mathrm{Ag}^{+}$and $\mathrm{Ni}^{2+}$. Thus, a high concentration of $\mathrm{Ag}^{+}$has a negative effect on the quality of powders. The experimental results show that if the amount of nucleating agent is controlled properly, these powders become not only well-dispersed but also are nano-sized.

\section{Summary and Conclusions}

Ethylene glycol was used in the production of Ni nanopowders in this study. A summary and conclusions of our findings follows.

(1) Nickel nanopowders, with a crystal size of $\sim 20 \mathrm{~nm}$ and an agglomeration size of $100-200 \mathrm{~nm}$, were successfully prepared in an ethylene glycol-hydrazine-ammonia (EHA) system.

(2) Ethylene glycol was found to be effective in preventing the nanopowders from agglomerating.

(3) The powder size decreased with the increasing amounts of hydrazine used and a nucleation agent, $\mathrm{AgNO}_{3}$, was found to be essential in promoting the formation of nickel powders.

\section{Acknowledgements}

Authors wish to acknowledge the financial support provided under contract \#M10212430001-02B1543-00210 by KISTEP (Korean Inst. of Sci. Tech. Evaluation and Planning) through the National R\&D Project for Nano Science and Technology. Also, we thank to Mr. Sangho Park in the preparation of manuscript.

\section{REFERENCES}

1) D. Ai and S. Kang: Ceramics Int. 30 (2004) 619-623.

2) H. D. Glicksman: Materials handbook. 7, $9^{\text {th }}$ ed. (Ameriacan Society for metals park, OH, 1984) 147-151.

3) C. Wang, X. M. Zhang and X. F. Qian: Mater. Res. Bull. 33 (1988) 1747-1751.

4) K. Yurij, F. Asuncion, R. T. Cristina, C. Juan, P. Pilar, P. Ruslan and G. Aharon: Chem. Mater. 11 (1999) 1331-1334.

5) S. Illy, O. Tillement, F. Machizaud and J. M. Dubois: Phyilos. Mag. A 79 (1999) 1021-1025.

6) S. Komarneni, R. Pidugu, Q. H. Li and R. Roy: J. Mater. Res. 10 (1995) 1687-1689.

7) E. G. Baburaj, K. T. Hubert and F. H. Froes: J. Alloy. Comp. 257 (1997) 146-149.

8) M. S. Hegde, D. Larcher and L. Dupont: Solid State Ioni. 93 (1997) 3350.

9) Y. D. Li, C. W. Li and H. R. Wang: Mater. Chem. Phys. 59 (1999) 8890.

10) J. Gao, F. Guan and Y. Zhao: Mater. Chem. Phys. 71 (2001) 215-219.

11) J. S. Jung: J. Phys. Chem. Solids 64 (2003) 385-390.

12) A. Degen and J. Macek: Nanostructured Mater. 12 (1999) 225-228.

13) Syukri, T. Ban, Y. Ohya and Y. Takahashi: Mater. Chem. Phys. 78 (2003) 645-649.

14) T. Hinotsu, B. Jeyadevan, C. N. Chinnasamy, K. Shinoda and K. Tohji: J. Appl. Phys. 95 (2004) 7477-7479.

15) C. M. Liu, L. Guo, R. M. Wang, Y. Deng, H. B. Xu and S. Yang: Chem. Commun. (2004) 2726-2727.

16) G. Viau: Solid State Ioni. 84 (1996) 259-270.

17) T. Sugimoto: Adv. Colloid Interface Sci. 28 (1987) 65-108.

18) S. Bhattacharjee: Croatica Chem. Acta. 71 (1988) 883-903. 